\title{
24-Hour Levodopa-Carbidopa Intestinal Gel: Clinical Experience and Practical Recommendations
}

\author{
Sandeep Thakkar ${ }^{1} \cdot$ Victor S. C. Fung $^{2} \cdot$ Aristide Merola $^{3} \cdot$ Meredith Rollins $^{4} \cdot$ Michael J. Soileau ${ }^{4} \cdot$ Norbert Kovács $^{5,6}$
}

Accepted: 5 December 2020 / Published online: 13 February 2021

(c) The Author(s) 2021

\begin{abstract}
Infusion of levodopa-carbidopa intestinal gel (LCIG; also designated carbidopa-levodopa enteral suspension) for 16 hours is a standard treatment for patients with advanced Parkinson's disease, and clinical observations suggest that 24-hour LCIG infusion may further reduce symptoms. This review provides practical advice on the management of patients transitioning to 24-hour LCIG infusion. We review available clinical data for 24-hour infusion and discuss adjustments to dosing, recommendations for monitoring, and management of patient concerns, based on our clinical experience. Data from multiple studies suggest that LCIG may improve non-motor symptoms. Although few studies have examined 24-hour LCIG infusion, available data indicate that certain patients may benefit from around-the-clock treatment. Studies of 24-hour LCIG infusion are limited by small sample sizes and open-label study designs, which may hamper translation to clinical practice. In our experience, we have found that patients may benefit from 24-hour infusion when reductions in nocturnal symptoms and improvements to quality of sleep are needed. Levodopa-unresponsive freezing of gait or poorly controlled troublesome dyskinesias may also indicate a patient may benefit from 24-hour infusion. Dose adjustments, especially of the nocturnal rate, are typically necessary and, as with 16-hour infusion, patients should be monitored for autonomic dysfunction; overnight wearing off symptoms; weight changes; fluctuations in plasma levels of vitamins $\mathrm{B}_{6} / \mathrm{B}_{12}$, folate, and homocysteine; changes in sleep patterns; or worsening of hallucinations, delusions, and/or nightmares. Available data and our clinical experience suggest that 24-hour LCIG may be warranted among selected patients who have poorly controlled nocturnal fluctuations or early morning "off" symptoms.
\end{abstract}

Sandeep Thakkar

sandeep.thakkar@Hoag.org

1 Department of Neuroscience, Hoag Memorial Hospital Presbyterian, 510 Superior Ave, Suite 200A, Newport Beach, CA 92663, USA

2 Movement Disorders Unit, Department of Neurology, Westmead Hospital and Sydney Medical School, University of Sydney, Sydney, NSW, Australia

3 Department of Neurology, The Ohio State University Wexner Medical Center, Columbus, OH, USA

4 Texas Movement Disorder Specialists, Georgetown, TX, USA

5 Department of Neurology, Medical School, University of Pécs, Pécs, Hungary

6 MTA-PTE Clinical Neuroimaging MR Research Group, Pécs, Hungary

\section{Key Points}

Infusion of levodopa-carbidopa intestinal gel for 16 hours is a standard treatment for patients with advanced Parkinson's disease

Certain patients may benefit from 24-hour levodopa-carbidopa intestinal gel infusion, particularly if reductions in nocturnal symptoms and improvements to quality of sleep are needed

Transitioning to 24-hour infusion typically requires dose adjustments and careful monitoring of patients 


\section{Introduction}

Parkinson's disease (PD) is a progressive neurodegenerative movement disorder characterized by motor and non-motor symptoms (NMS). The gold standard for treatment of PD motor symptoms is oral levodopa $[1,2]$. With PD progression, the therapeutic window needed to achieve optimal motor control narrows, giving the appearance of waning levodopa efficacy [1]. Oral dosing may lead to fluctuating plasma levodopa concentrations, caused by the drug's short half-life and erratic intestinal absorption [2]. Intermittent/ pulsatile dopamine receptor stimulation ultimately leads to worsening motor and NMS, which represent severe impairments to quality of life in patients with advanced PD [2, 3]. In particular, NMS represent a considerable source of functional disability in PD, and some patients report NMS to be even more bothersome than motor fluctuations $[4,5]$. The effects of fluctuating plasma levodopa concentrations can be mitigated using continuous dopaminergic stimulation to maintain plasma levodopa concentrations within a therapeutic window $[2,3]$.

\section{Levodopa-Carbidopa Intestinal Gel}

\subsection{Overview}

Levodopa-carbidopa intestinal gel (LCIG; designated in the USA as carbidopa-levodopa enteral suspension) is an approved treatment for advanced PD that continuously delivers levodopa through infusion into the proximal jejunum via a percutaneous endoscopic gastrostomy tube with a jejunal extension (PEG-J) connected to a portable infusion pump. Efficacy of up to 16-hours continuous administration of LCIG in reducing motor fluctuations has been established in multiple prospective studies [6-18], and reviewed extensively [2, 19-21]. Although the pathogenesis of NMS remains partially unclear, 16-hour (16-h) continuous administration of LCIG has been associated with improvements in NMS, sleep quality, and quality-of-life measures (Table 1) $[6,7,11,13,17,22,23]$.

\subsection{Effects of LCIG on Non-Motor Symptoms}

Reductions in plasma levodopa fluctuations have been shown to decrease NMS. Data from the GLORIA registry demonstrated improvements in patient quality of life and reductions in NMS, including sleep and fatigue [6, 7]. In addition, baseline NMS burden was associated with improvements in NMS and quality of life [22]. Similarly, in the GREENFIELD observational cohort, significant improvements were observed in quality of life, NMS, and sleep [13]. Standaert et al. [17] observed reductions in mean hours of "off" time and increases in "on" time without troublesome dyskinesia through 60 weeks of LCIG treatment, along with improvements in NMS and quality-of-life measures. A Hungarian LCIG registry demonstrated that 12-month LCIG treatment reduced dyskinesia severity and disability and improved NMS, quality of life, and sleep [11]. Polysomnographic data of LCIG-treated patients indicated reductions in the number of night-time awakenings, but not changes in total sleep time, sleep efficiency/latency, rapid eye movement, or wake time after sleep onset [23]. The improvements to sleep observed in these studies of 16-h infusion of LCIG indicate that reductions in daytime "off" periods may be beneficial to night-time symptoms. Collectively, these data suggest that 16-h infusion of LCIG may alleviate NMS burden and sleep problems experienced by patients with advanced PD.

\subsection{Efficacy of 24-Hour LCIG Use}

Further reductions in motor and NMS have been observed in some patients treated with LCIG around the clock. We have found that symptoms related to wearing off of levodopa overnight (e.g., insomnia, stiffness, tremor, and dystonia) are the main issues leading to a patient's transition to 24-hour (24-h) LCIG. Although the mechanism of action is not well understood, the observed additional benefits of 24-h LCIG use are thought to be the result of pharmacodynamic processes that rely on steady and continuous delivery of levodopa to the brain [24]. Efficacy of 24-h LCIG infusion has been reported in several case series and small clinical studies and are discussed below (Table 2). We identified these reports through PubMed searches, using the search terms "24 hour", "levodopa carbidopa intestinal gel", "Duodopa", and "Duopa". Bibliographies of reports identified in PubMed searches were also examined for other relevant references. All reports identified in this manner are discussed herein.

In a retrospective case series of five male patients who received 24-h LCIG infusion for $\leq 37$ months (range 13-37 months) for the treatment of motor fluctuations and nocturnal akinesia, Nyholm et al. [25] observed improvements in motor function and sleep, without development of clinically relevant levodopa tolerance or increases in hallucinations or dyskinesia. In another case series of three patients who received 24-h LCIG infusion, all reported good effects on severe nocturnal dystonic pain, two experienced reduced motor symptoms, and one experienced increased motor symptoms after 1 month [26].

Improved sleep quality was observed in a retrospective study of 21 patients treated with 24-h LCIG [27]. Over the mean 32-month treatment time with 24-h LCIG, night-time doses averaged $76 \pm 18 \%$ of daytime infusion rates, and dosages increased by $15 \pm 24 \%$ in 14 of 21 patients [27]. 
Table 1 Summary of studies evaluating 16-hour (16-h) levodopa-carbidopa intestinal gel (LCIG) for non-motor symptoms

\begin{tabular}{|c|c|c|c|c|}
\hline Reference & $N$ & Follow-up time & Measure & Results \\
\hline \multirow[t]{2}{*}{ Standaert et al. 2017 [17] } & \multirow[t]{2}{*}{28} & \multirow[t]{2}{*}{60 weeks } & NMSS total score & $\begin{array}{l}\text { LS mean (SE) change from baseline to week } 60 \text { : } \\
-11.8(4.0), p=0.004\end{array}$ \\
\hline & & & PDQ-39 & $\begin{array}{l}\text { LS mean (SE) change from baseline to week } 60 \text { : } \\
-10.2(2.6), p<0.001\end{array}$ \\
\hline \multirow[t]{3}{*}{$\begin{array}{l}\text { Antonini et al. } 2017 \\
\text { (GLORIA registry) [6] }\end{array}$} & \multirow[t]{3}{*}{375} & \multirow[t]{3}{*}{24 months } & NMSS total score & $\begin{array}{l}\text { Mean (SD) change from baseline to last study visit: } \\
-14.4(44.8) \text { [95\% CI }-20.3,-8.5], p<0.001\end{array}$ \\
\hline & & & NMSS sleep/fatigue & $\begin{array}{l}\text { Mean (SD) change from baseline to last study visit: } \\
-4.5(10.6)[95 \% \text { CI }-5.9,-3.1], p<0.001\end{array}$ \\
\hline & & & PDQ-8 & $\begin{array}{l}\text { Mean (SD) change from baseline to last study visit: } \\
-5.3(20.7)[95 \% \text { CI }-8.2,-2.5], p<0.001\end{array}$ \\
\hline \multirow[t]{3}{*}{ Juhasz et al. 2017 [11] } & \multirow[t]{3}{*}{34} & \multirow[t]{3}{*}{12 months } & $\begin{array}{l}\text { Number of patients with PDSS- } \\
2 \text { total score } \geq 11\end{array}$ & $\begin{array}{l}\text { Baseline, } 15 \text { patients; follow-up, } 7 \text { patients, } \\
p=0.021\end{array}$ \\
\hline & & & PDSS-2 total score & $\begin{array}{l}\text { Mean (SD) score: } \\
\text { baseline, } 27.2 \text { (10.5); follow-up, } 23.2 \text { (12.0), } \\
p=0.042\end{array}$ \\
\hline & & & PDQ-39 & $\begin{array}{l}\text { Mean (SD) score: } \\
\text { baseline, } 38.5 \text { (14.9); follow-up, } 29.6 \text { (13.6), } \\
p=0.003\end{array}$ \\
\hline \multirow[t]{5}{*}{ Zibetti et al. 2017 [23] } & \multirow[t]{5}{*}{11} & \multirow[t]{5}{*}{$3.8 \pm 1.2$ months } & Polysomnograph recording & $\begin{array}{l}\text { Mean (SD) awakenings in sleep: } \\
\text { baseline, } 10.5(12.2) \text {; follow-up, } 4.9 \text { (2.5), } \\
p=0.026\end{array}$ \\
\hline & & & PDSS-2 total score & $\begin{array}{l}\text { Mean (SD) score: } \\
\text { baseline, } 31.0(6.3) \text {; follow-up, } 18.0(8.9) \text {, } \\
p=0.046\end{array}$ \\
\hline & & & Disturbed sleep & $\begin{array}{l}\text { Mean (SD) score: } \\
\text { baseline, } 12.9 \text { (3.9); follow-up, } 7.2 \text { (2.6), } \\
p=0.041\end{array}$ \\
\hline & & & Motor symptoms at night & $\begin{array}{l}\text { Mean (SD) score: } \\
\text { baseline, } 10.4 \text { (3.7); follow-up, } 5.5 \text { (4.2), } \\
p=0.043\end{array}$ \\
\hline & & & PD symptoms at night & $\begin{array}{l}\text { Mean (SD) score: } \\
\text { baseline, } 8.0 \text { (3.7); follow-up, } 5.3(4.1) \text {, } \\
p=0.027\end{array}$ \\
\hline \multirow[t]{2}{*}{$\begin{array}{l}\text { Lopiano et al. } 2019 \\
\text { (GREENFIELD cohort) [13] }\end{array}$} & \multirow[t]{2}{*}{145} & \multirow[t]{2}{*}{ Mean 3 years } & PDSS-2 & $\begin{array}{l}\text { Mean (SD) score: } \\
\text { baseline, } 25 \text { (10.4); follow-up, } 22.5 \text { (9.9), } \\
p<0.01\end{array}$ \\
\hline & & & PDQ-39 & $\begin{array}{l}\text { Mean (SD) score: } \\
\text { baseline, } 72.3 \text { (23.8); follow-up, } 67.3 \text { (26.4), } \\
p<0.05\end{array}$ \\
\hline
\end{tabular}

All studies reviewed in Table 1 examined 16-h LCIG use

$C I$ confidence interval, $L S$ least squares, NMSS Non-Motor Symptom Scale, $P D$ Parkinson's disease, $P D Q$ Parkinson's Disease Questionnaire, $P D S S$-2 Parkinson's Disease Sleep Scale, SD standard deviation, $S E$ standard error

Chang et al. [28] conducted a prospective open-label study of 24-h LCIG infusion as a treatment for unresponsive freezing of gait (u-FOG) and falls prevention. Four patients receiving 16-h LCIG infusion and one patient with disabling u-FOG who was receiving oral levodopa were transitioned to 24-h LCIG, with night-time infusion rates that were 50-80\% of the typical daytime rate [28]. Reductions in $360^{\circ}$ turning time and median falls frequency, along with improvements on the FOG questionnaire, were observed at 3 and
6 months, although no changes in timed up-and-go 8-minute walk times were observed [28].

Ricciardi et al. [29] evaluated eight patients who, at last follow-up, had received 24-h treatment for $26 \pm 31.6$ months (range, 4-72 months). In seven of eight patients, the infusion rate was reduced at night-time by a mean of $27 \%$, with no signs that patients were developing a tolerance to treatment [29]. Significant improvements from baseline were observed for NMS, specifically in sleep/fatigue, mood/ 
Table 2 Summary of studies evaluating 24-hour (24-h) levodopa-carbidopa intestinal gel (LCIG) infusion

\begin{tabular}{|c|c|c|c|c|}
\hline Reference & $N$ & Follow-up time & Measure & Results \\
\hline Nyholm et al. 2005 [25] & 5 & Up to 37 months & Clinical observation & $\begin{array}{l}\text { Improvements in motor function and } \\
\text { sleep }\end{array}$ \\
\hline Karlsborg et al. 2010 [26] & 3 & 1 month & UPDRS III & $\begin{array}{l}\text { Two patients experienced reductions in } \\
\text { score ( } 35 \% \text { and } 48 \% \text { ), while } 1 \text { patient } \\
\text { experienced a } 50 \% \text { increase }\end{array}$ \\
\hline Busk and Nyholm 2012 [27] & 21 & $32 \pm 28$ months & PDSS-2 & $\begin{array}{l}\text { Median (range) before } 24-\mathrm{h} \text { treatment, } 3 \\
(2-4) \text {; during } 24-\mathrm{h} \text { treatment, } 1(0-4) \\
p<0.001\end{array}$ \\
\hline \multirow[t]{4}{*}{ Chang et al. 2015 [28] } & \multirow[t]{4}{*}{5} & \multirow[t]{4}{*}{6 months } & Timed up-and-go 8-minute walk & $\begin{array}{l}\text { Median (SD) at baseline, } 7.78(30.4) \mathrm{s} \text {; at } \\
\text { follow-up, } 8.03(4.9) \mathrm{s}\end{array}$ \\
\hline & & & $360^{\circ}$ turning time & $\begin{array}{l}\text { Median (SD) at baseline, } 15.47(7.06) \mathrm{s} \\
\text { at follow-up, } 8.27(14.72) \mathrm{s}\end{array}$ \\
\hline & & & FOG questionnaire & $\begin{array}{l}\text { Median (SD) at baseline, } 18.0 \text { (4.6); at } \\
\text { follow-up, } 15.5(5.2)\end{array}$ \\
\hline & & & Falls frequency & $\begin{array}{l}\text { Median score at baseline, } 4 \text { (daily); at } \\
\text { follow-up, } 0 \text { (none) }\end{array}$ \\
\hline \multirow[t]{5}{*}{ Ricciardi et al. 2016 [29] } & \multirow[t]{5}{*}{8} & \multirow[t]{5}{*}{$26 \pm 31.6$ months } & NMSS sleep/fatigue & $\begin{array}{l}\text { Mean (SD) at baseline, } 19.3(11.2) \text {; at } \\
\text { follow-up, } 9.5(6.6), p=0.02\end{array}$ \\
\hline & & & NMSS mood/cognition & $\begin{array}{l}\text { Mean (SD) at baseline, } 28.8(24.7) \text {; at } \\
\text { follow-up, } 8.7(8.6), p=0.04\end{array}$ \\
\hline & & & NMSS hallucination & $\begin{array}{l}\text { Mean (SD) at baseline, } 8.8(9.4) \text {; at } \\
\text { follow-up, } 4.2(5.5), p=0.04\end{array}$ \\
\hline & & & NMSS urinary symptoms & $\begin{array}{l}\text { Mean (SD) at baseline, } 15(7.6) \text {; at } \\
\text { follow-up, } 4.6(4.3), p=0.04\end{array}$ \\
\hline & & & PDSS & $\begin{array}{l}\text { Mean (SD) at baseline, } 39(11.7) \text {; at } \\
\text { follow-up, } 31.1(14.3), p=0.02\end{array}$ \\
\hline \multirow[t]{2}{*}{ Cruse et al. 2018 [24] } & \multirow[t]{2}{*}{12} & \multirow[t]{2}{*}{ Median 27.5 months } & $\begin{array}{l}\text { UPDRS IV item } 32 \text { (time spent with } \\
\text { dyskinesia) }\end{array}$ & Mean (SD) change, $-1.5(0.75), p=0.01$ \\
\hline & & & $\begin{array}{l}\text { UPDRS IV item } 33 \text { (functional impact } \\
\text { of dyskinesia) }\end{array}$ & $\begin{array}{l}\text { Mean }(\mathrm{SD}) \text { change, }-1.7(0.90), p= \\
0.016\end{array}$ \\
\hline \multirow[t]{2}{*}{ Morales-Briceño et al. 2019 [30] } & \multirow[t]{2}{*}{35} & \multirow[t]{2}{*}{$11 \pm 2$ months } & UPDRS IV (total) & $\begin{array}{l}\text { 16-h LCIG mean (SD), } 9.3(4.0) \\
\text { 24-h LCIG mean (SD), } 7.0(3.0) \\
p=0.001\end{array}$ \\
\hline & & & $\begin{array}{l}\text { UPDRS IV item } 36 \text { (complexity of } \\
\text { motor fluctuations subscore) }\end{array}$ & $\begin{array}{l}\text { 16-h LCIG mean (SD), } 2.2(0.6) \\
\text { 24-h LCIG mean (SD), } 1.5(0.5) \\
p=0.002\end{array}$ \\
\hline Nyholm et al. 2020 [31] & 7 & N/A & LCIG pump data & $\begin{array}{l}\text { Median extra dose, } 24-\mathrm{h} \text { LCIG, } 32 \mathrm{mg} \\
\text { (range: } 0-82 \mathrm{mg} \text { ) } \\
\text { Median extra dose, 16-h LCIG, } 20 \mathrm{mg} \\
\text { (range: } 6-40 \mathrm{mg} \text { ) }\end{array}$ \\
\hline
\end{tabular}

16- $h 16$ hour, FOG freezing of gait, N/A not available, NMSS Non-Motor Symptom Scale, PDSS-2 Parkinson's Disease Sleep Scale, SD standard deviation, UPDRS Unified Parkinson's Disease Rating Scale

cognition, hallucination, urinary problems, and sleep quality [29]. These overall improvements in sleep quality, daytime sleepiness, and fatigue are consistent with improvements in nocturnal akinesia [29].

Cruse et al. [24] evaluated use of 24-h LCIG for dyskinesia in a retrospective analysis of 12 patients with median follow-up time of 27.5 months. Patients were transitioned to 24-h LCIG because of troublesome dyskinesia alone and lack of response to 16-h LCIG $(n=4)$, troublesome dyskinesia and levodopa u-FOG $(n=2)$, levodopa u-FOG with non-troublesome dyskinesia $(n=3)$, troublesome dyskinesia and nocturnal akinesia $(n=2)$, and patient-initiated 24-h use $(n=1)$ [24]. After transition to 24-h LCIG, nine patients reported reduced dyskinesia and two reported improved FOG [24]. Reductions in time spent with dyskinesia and functional impact of dyskinesia were observed, without change to the Unified Parkinson Disease Rating Scale III "on" scores; worsening of dyskinesia following transition to 24-h LCIG was not observed in any patients [24].

Most recently, Morales-Briceño et al. [30] reported longterm safety and efficacy of 35 patients treated with 24-h LCIG. Patients were either switched from 16-h LCIG $(n=$ 
28 ) or started directly on 24-h LCIG from oral dopaminergic and/or apomorphine treatment $(n=7)$ [30]. The safety profile was not significantly different between $24-\mathrm{h}$ and $16-\mathrm{h}$ LCIG infusion, except for a significantly lower incidence of neuropathy with 24-h infusion ( $p=0.02$ ) [30]. Significant reductions in total Unified Parkinson Disease Rating Scale Part IV $(p=0.001)$ and the complexity of motor fluctuations subscore $(p=0.002)$ were observed after $11 \pm 2$ months of treatment with $24-\mathrm{h}$ vs $16-\mathrm{h}$ LCIG [30].

Nyholm et al. [31] analyzed pump data obtained from 25 LCIG-treated patients ( $n=7,24-\mathrm{h} \mathrm{LCIG)}$ to determine realworld patterns of extra dose usage among daytime and 24-h LCIG users. The median daily levodopa dose among the 24-h LCIG-treated patients was $1928 \mathrm{mg}$ (range 940-2180 $\mathrm{mg}$ ) [31]. None of the patients receiving 24-h LCIG used a morning dose [31]. Compared with the 16-h LCIG group, the 24-h patients used more (mean number of extra doses per day, 24 hours, $3.6 \pm 3.5$; 16 hours, $2.8 \pm 2.2$ ) and higher extra doses [median (range) extra dose, 24 hours, $32 \mathrm{mg}$ (0-82 mg); 16 hours, $20 \mathrm{mg}$ (6-40 mg)] [31]. The authors note that a high number of extra doses may be indicative of a too-low continuous dose, or that the patient overuses the extra dose button [31].

\section{Practical Experience with 24-h LCIG Infusion}

Currently available data offer evidence that $24-\mathrm{h}$ infusion of LCIG may provide additional reductions in symptoms beyond those apparent with daytime LCIG infusion. However, these studies are limited by small sample sizes, openlabel study designs, and a lack of control groups. Larger studies with appropriate control groups are needed to confirm these findings. At present, 24-h LCIG infusion is offlabel in the USA and only indicated when medically necessary in the European Union. Regardless, 24-h LCIG infusion is used in clinical practice, and given the limits of current evidence, practical advice on patient management may be of value. Detailed guidance on administration and management of 16-h LCIG infusion has recently been published [32], but, to our knowledge, no similar guide exists for 24-h LCIG infusion. Here, we present our suggestions for transitioning to and treating patients with 24-h LCIG infusion.

We specialize in treating patients with movement disorders using both 16- and 24-h LCIG infusion. At our clinical practices, the number of patients who have been switched to 24-h LCIG administration varies, with the total percent of patients treated with 24-h LCIG ranging from 5 to $40 \%$ (range 5-100 patients: total of 200 patients between practices). In addition, some of our patients have been treated with deep brain stimulation (DBS) in conjunction with 24-h LCIG (range 1-18 patients between practices). Both levodopa and DBS improve motor symptoms independently, and results from a recent meta-analysis indicate that they may have a synergistic effect [33]. However, in some patients treated with DBS, it is impossible to harness the full potential of brain stimulation because of trade-offs between clinical benefit and stimulation-induced side effects. Furthermore, most patients who receive DBS still require levodopa in some form to manage symptoms. In particular, symptoms such as freezing, balance, mood, and fatigue are difficult to treat with DBS, but are often addressed with LCIG. Regardless of whether a patient receives DBS as PD progresses, the therapeutic window continues to narrow and patients will experience motor fluctuations. Therefore, some patients may still be candidates for 24-h LCIG infusion, whether or not they also receive DBS. We pooled our experiences with 24-h administration of LCIG, with the goal of providing practical advice on the use of LCIG for 24-h continuous dosing in patients with advanced PD (Table 3).

\section{Clinical Perspective}

\subsection{Patients Who May Benefit From 24-h LCIG Use}

The primary goal of 24-h LCIG treatment is to reduce nocturnal fluctuations in motor symptoms and NMS and improve quality of sleep. Patients may benefit from 24-h LCIG administration if they experience noteworthy wearing off of symptoms overnight, delayed morning latency (i.e., delayed time to "on" following the first morning levodopa dose) [34], insomnia, stiffness, tremor, or dystonia due to wearing off of symptoms overnight, severe night-time akinesia or bradykinesia, severe night-time pain, and/or severe night-time urinary problems. Because of the limited evidence regarding 24-h LCIG infusion, the initiation of 24-h treatment should be decided individually, after weighing the potential benefits and drawbacks. Each patient is unique, and decisions regarding initiation of 24-h infusion should generally be based on whether other options for night-time symptom control have failed or are inappropriate. For instance, alternatives of dopamine agonists (e.g., rotigotine transdermal patch) may exacerbate problems such as neurogenic orthostatic hypotension or hallucinations. As another example, some of our patients have reported experiencing severe "off" time during the night despite taking oral carbidopa/ levodopa extended release at bedtime and carbidopa/levodopa immediate release every 2 hours during the night. As a result, these patients are unable to sleep for more than a few hours at a time because of muscle cramps and other "off" symptoms. Many patients with advanced PD also experience rigidity and gait imbalance at night, and we have found continuous infusion to reduce these symptoms. Additionally, some patients treated with 16-h LCIG turn on the pump at 
Table 3 Summary of key points and practical advice

\begin{tabular}{|c|c|}
\hline Key point & Clinical tips \\
\hline Patients who may benefit from 24-h LCIG treatment & $\begin{array}{l}\text { Severe nocturnal motor and non-motor symptoms despite use of other } \\
\text { oral medications } \\
\text { Disabling dyskinesia related to flushing the tube before stopping the } \\
\text { LCIG pump } \\
\text { Severe or disabling early morning "off" symptoms } \\
\text { Significant caregiver burden at night-time }\end{array}$ \\
\hline $\begin{array}{l}\text { Dose adjustments, particularly to the morning dose, are typically } \\
\text { needed when patients switch to 24-h LCIG administration }\end{array}$ & $\begin{array}{l}\text { Infusion rates may initially be lowered by } 80 \% \text { of the } 16 \text {-hour dose; } \\
\text { titrating as needed to reach the optimal rate for symptom control } \\
\text { The morning dose should be reduced or eliminated, depending on } \\
\text { wake-up time or morning symptoms } \\
\text { Patients should be encouraged to use extra doses during periods of } \\
\text { predictable "off" time }\end{array}$ \\
\hline $\begin{array}{l}\text { Patients receiving } 24-\mathrm{h} \text { LCIG administration should be monitored } \\
\text { routinely for side effects or effects on sleep }\end{array}$ & $\begin{array}{l}\text { Blood pressure may be checked every morning, especially if there is } \\
\text { concern for neurogenic orthostatic hypotension } \\
\text { Similar to 16-hour infusion, potential concerns include: } \\
\text { - Weight loss } \\
\text { - Development of polyneuropathies } \\
\text { - Effects on sexual activity } \\
\text { - Development or worsening of pre-existing impulse control disorders } \\
\text { - Excessive night-time eating } \\
\text { - Exacerbation of hallucinations, delusions, or nightmares } \\
\text { Routinely monitor patients for changes to plasma levels of vitamins } \mathrm{B}_{6} \\
\text { and } \mathrm{B}_{12} \text {, folate, and homocysteine } \\
\text { Monitor patients for symptoms of LCIG wearing off overnight and } \\
\text { changes to sleep patterns } \\
\text { A lower infusion dose may be used if dyskinesia that interferes with } \\
\text { sleep quality occurs } \\
\text { If visual hallucinations are exacerbated, quetiapine, clozapine, or pima- } \\
\text { vanserin (not available in the European Union) may be initiated }\end{array}$ \\
\hline Patients should be reminded to regularly flush the tubing & $\begin{array}{l}\text { Flushing recommendations include: } \\
\text { - Every morning, prior to the morning dose } \\
\text { - Whenever the cassette is changed } \\
\text { - If the infusion is stopped for } 1 \text { hour each day, can recommend flush- } \\
\text { ing immediately after disconnecting } \\
\text { - Recommend flushing twice per day with carbonated water, rather } \\
\text { than water/saline } \\
\text { Recommend that the tubing be changed every } 1-2 \text { years }\end{array}$ \\
\hline
\end{tabular}

24-h 24 hour, LCIG levodopa-carbidopa intestinal gel

bedtime to alleviate night-time symptoms that prevent them from falling or staying asleep.

Another situation we have encountered is that, when flushing the tube before bedtime, some patients may experience dyskinesia because of an excessive intake of levodopa contained in the tube, which may cause physical (e.g., dyskinesia) and mental (e.g., confusion, hallucinations) problems that may interfere with the first phase of sleep. These patients may be good candidates for 24-h LCIG infusion. Some patients switch to 24-h LCIG infusion with disease progression, often 6 months to 1 year after initiating 16-h LCIG infusion. Patients may also desire early morning "on" time, which may be facilitated by $24-\mathrm{h}$ infusion. Finally, when reducing patient and caregiver burden is desired, 24-h LCIG administration may be useful, as it lessens the need for caregivers to assist with tasks such as toileting and medication administration. In addition, if wearing off does not occur, the risk of night-time falls may be reduced.

Some patients undergoing 16-h LCIG infusion may resist switching back to oral levodopa at night, and we have encountered patients who insist on infusion around the clock to ameliorate their symptoms. However, use of 24-h LCIG dosing depends on a patient's therapeutic window, as a narrower window entails a greater need for continuous levodopa. Another potential consideration involves technical aspects of the LCIG pump itself. The currently available pump lacks a clock and an alarm to indicate when the cassette requires replacement. While some apomorphine pumps may be programmed with several different infusion rates or for automatic or manual switches between rates, the LCIG pump lacks this feature. Patients and caregivers must therefore have the cognitive and motor skills to program the pump 
for 24-h use independently, to avoid any potential problems or overdosing. The decision to switch a patient to 24-h LCIG infusion is therefore highly patient specific.

\subsection{Strategies To Consider Before Starting 24-h LCIG}

Before initiating 24-h LCIG infusion for their patients, providers may wish to consider other strategies for symptom control, some of which include off-label use of certain medications. It is generally preferable to initiate daytime $16-\mathrm{h}$ infusion of LCIG, rather than immediately transition patients from oral levodopa to 24-h LCIG infusion. For patients receiving 16-h LCIG, providers may recommend using extra doses when stopping the pump and flushing in the evening, before transitioning to 24-h LCIG use. This strategy also provides a dosing boost from flushing the medication that remains in the tubing. Furthermore, if daytime medications do not control symptoms and/or side effects, other methods for supplementing dopamine with strategic timing can be useful. Immediate- or extended-release formulations of dopamine agonists, such as the rotigotine patch, ropinirole, or pramipexole, may be prescribed to control night-time symptoms. Some providers may prefer the rotigotine patch, as it can be removed in the morning, facilitating precise titration of the overnight dose without affecting the daytime rate. Immediate-release ropinirole, pramipexole, and gabapentin may be especially useful when patients report symptoms of restless leg syndrome. Slow-release oral levodopa or levodopa/carbidopa/entacapone may be tried before administering a dopamine agonist, or if dopamine agonists are insufficient, not well tolerated, or otherwise contraindicated. Some providers may not prefer dopamine agonists for patients with advanced PD because adverse effects such as hallucinations or neurogenic orthostatic hypertension may occur. If all these options fail, 24-h LCIG administration may be appropriate.

Some providers may wish to prescribe 24-h infusion for a brief period to evaluate any improvements or curtail worsening of side effects. One method is to switch patients from 16-h to 24-h LCIG infusion for a trial period of approximately 2 weeks. Another approach is to increase the infusion time from 16 hours to 18 hours for a few days, and then fully transition patients to 24-h LCIG use. However, some patients do not adhere strictly to the 16-h limit, instead wearing the pump during waking hours and unhooking it at bedtime. For these patients, the latter strategy may need to be modified to accommodate patients' usual schedule, or a different strategy may be implemented.

\subsection{Adjustments to Dosing}

Adjustments to the morning and/or continuous dose are often required when switching patients to 24-h LCIG infusion. Our approaches vary, and the adjustments are likely to depend on provider preference, although, whenever possible, dosing should be kept convenient for patients. Each LCIG cassette contains approximately $2000 \mathrm{mg}$ of levodopa. Although use of one cassette for more than 16 hours is not presently recommended by the current US Food and Drug Administration label, in clinical practice, the number of cassettes required for 24-h infusion will depend on patient needs. With 24-h LCIG infusion, some patients will not require more than $2000 \mathrm{mg} / \mathrm{day}$, and use of a second cassette may be avoided. Some of us (A.M. and N.K.) prefer to use only one cassette per day, if possible, as, in our clinical experience, a maximum dose of $2000 \mathrm{mg}$ /day may help to avoid neuropsychiatric complications such as hallucinations and dopaminergic dysregulation syndrome. Another consideration is that some countries allow reimbursement for only one cassette per day, which may result in increased outof-pocket costs for some patients. Such cost considerations must also be factored in when determining the appropriate number of cassettes. Ultimately, the dose required to control symptoms, and hence the number of cassettes needed over the 24-h period, is patient specific. The timing of the cassette change may also be discussed with patients. Some providers recommend that the cassette be changed at bedtime, while others recommend changing the cassette in the morning. A benefit of always changing the cassette at bedtime allows for five extra doses to be administered if needed, which may be helpful if symptoms of wearing off occur. The appropriate time to change the cassette will often depend on patient/ caregiver preference.

The dose should be optimized according to patient observation. When any adjustments are made, patients should be asked to keep a motor diary. This diary should count all extra doses administered so that the amount can be rolled back into the continuous rate. Initially, the night-time infusion rate may be reduced to $80 \%$ of the 16 -h rate, titrating as needed to reach the optimal rate for symptom control (Fig. 1). In some individual cases, a constant infusion dose may be maintained over the 24-h period, and the dose may be lowered to avoid exceeding $2000 \mathrm{mg} / \mathrm{day}$, if use of a single cassette alone is desired. For some patients, the daily infusion dose may be kept as low as possible, and providers can suggest using extra doses during periods of predictable "off" time (e.g., early afternoon). However, with this strategy, there is the possibility that dyskinesia may worsen, as many patients experience end-of-dose dyskinesia that may be exacerbated if the dose is low and close to the efficacy threshold. Another potential caveat is that use of extra doses may result in increased pulsatile stimulation that triggers peak-dose dyskinesia. Dose reductions may also be made only to the night-time dose, whereby night-time rates could be reduced by $50-80 \%$ of daytime rates. In the cohort of 35 patients reported by Morales-Briceño et al. [30], the average 


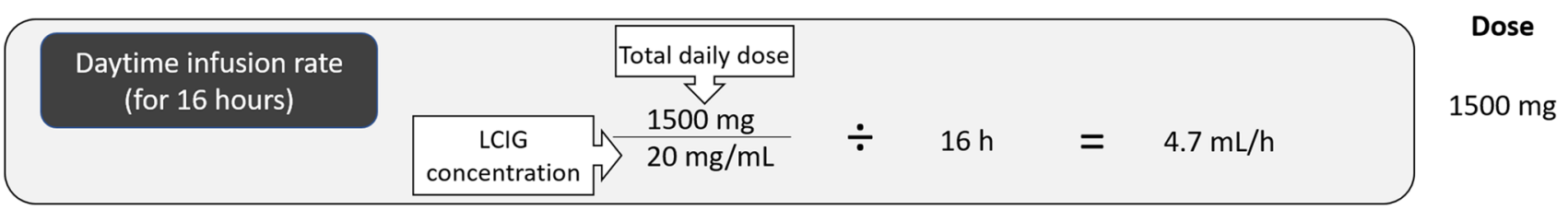

Example nighttime infusion rates (for 8 hours)

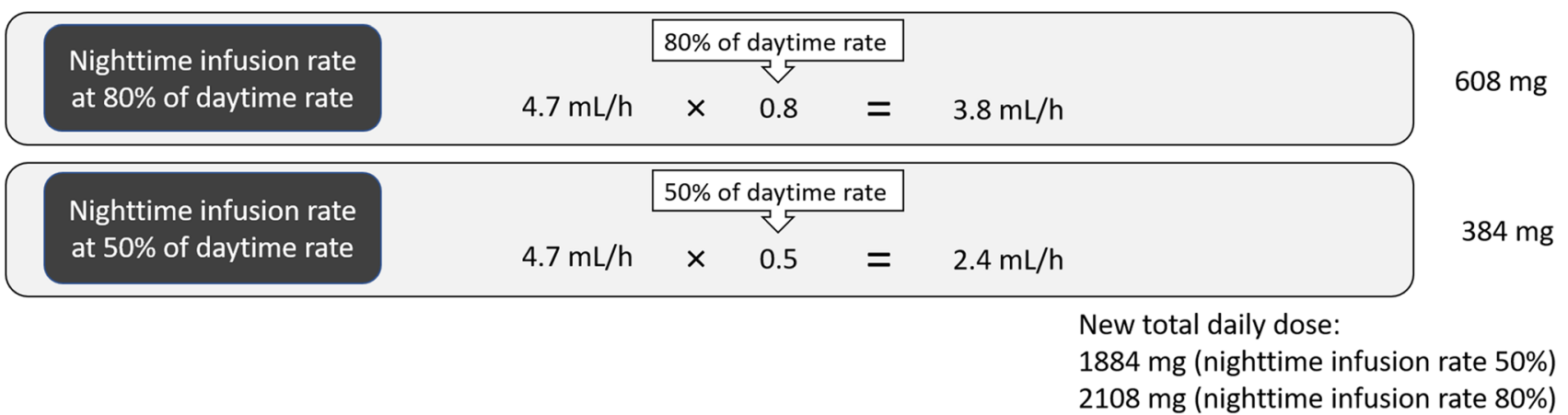

Fig. 1 Example of a patient with a daily total levodopa dose of $1500 \mathrm{mg}$, dosed over 16 hours, now requires 24-h dosing

nocturnal dose was approximately $60 \%$ of the daytime rate. The minimum night-time dose that improves night-time symptoms without eliciting dyskinesia, hallucinations, or other symptoms should be used. In their study of 24-h LCIG use, Cruse et al. [24] observed a wide range of night-time LCIG rates $(0.5-5.1 \mathrm{~mL} / \mathrm{h} ; 43-100 \%$ of the daytime rate) and suggest initially setting the night-time rate to $50 \%$ of the daytime rate. Then, titration to optimal dosing by $0.1 \mathrm{~mL} / \mathrm{h}$ of the night-time rate in 1- to 4-week intervals should be attempted [24]. Response of night-time symptoms to 24-h LCIG is typically patient or caregiver reported, although the PD Sleep Scale- 2 could be used to assess treatment effect on sleep.

The morning dose should be reduced/eliminated entirely, based on the patient's wake-up time and/or morning symptoms. For some patients (e.g., patients receiving a constant infusion rate per 24-h period), the morning dose can be eliminated. Other patients may require a reduced morning bolus. Alternately, the morning dose may be redistributed over the 24-h period. In cases where a lower night-time dose is given, a morning dose may still be needed. For some patients, early morning flushing of the tube may serve as a substitute for the morning dose. A general guideline is that if symptoms are severe, the morning dose is needed, but if symptoms are not severe, the nocturnal rate can be subtracted from the morning dose. Providers may also suggest that patients prime tubing with levodopa by giving a 3-mL morning bolus whenever the cassette is changed, which may be facilitated by changing the morning lockout time to 10 hours.

\subsection{Flushing Recommendations}

Patients should be reminded at every visit to regularly flush their tubing, although specific flushing recommendations vary between practitioners. Our recommendations for flushing vary between our practices, and include (1) flushing the tubing twice daily with carbonated water, rather than plain water or saline; (2) flushing whenever the cassette is changed; (3) flushing every day before the morning dose; and (4) flushing immediately after disconnecting the pump for 1 hour each morning and administering an extra dose to more quickly fill the tube when restarting the pump. The latter strategy may be helpful for patients who develop marked dyskinesia after flushing. When restarting the pump, a small bolus may be administered to limit drops in blood pressure.

Provider discretion and patient needs will dictate the flushing recommendations made to patients. For optimal treatment, flushing should occur at least once daily to prevent obstructions. Although it is unlikely that the tubing will become obstructed with 24-h infusion, no data have currently been reported with respect to the rate of obstruction without flushing. Therefore, we believe it preferable to flush daily, which can serve as a check to ensure that medication is flowing properly and that the $\mathrm{J}$ portion of the tube is not kinked. Furthermore, difficulty in flushing may be the patient's first indication that an obstruction or other problem is present. If flushing does not take place, the patient may not be aware of issues until the pump's high-pressure alarm sounds and/or their medication is no longer being 
effectively delivered. Flushing can thus act as an indicator that the patient should follow up with their proceduralist. In addition, in our experience [35-37], tubing should be changed approximately every 1.5 years to prevent the risk of dehiscence or other gastrointestinal complications, although replacement times may vary because of patient circumstances and provider preferences. We have observed that many gastroenterologists recommend this frequency, though others only recommend changing the tubing if there is a blockage or the J-tube is not in place.

\subsection{Monitoring and Follow-up Care}

In our practices, patient/caregiver education is typically provided by the nursing and advanced practice provider teams. Routine monitoring for patients treated with 24-h LCIG is generally the same as for those treated with 16-h LCIG as there are no unique concerns regarding stoma or tubing care, and the infection risk is not increased with 24-h infusion. We recommend scheduling a follow-up visit $1-2$ weeks after switching to 24-h use to discuss dose adjustments, side effects, and any other patient concerns.

Patients should be regularly monitored for levodopainduced side effects, particularly autonomic dysfunctions. Recent data indicate that the prevalence of dysautonomia in patients with advanced PD who are treated with LCIG approaches 50\% [38]. It is possible that constant levodopa infusion may minimize cardiovascular autonomic complications associated with dopamine agonists and enzymatic inhibitors. Although stable delivery of levodopa is likely to avoid the plasma peaks that may result in orthostatic hypotension, we recommend that blood pressure be monitored every morning, as patients may experience low blood pressure stemming from too much levodopa during the night. Although fluctuations in blood pressure are multifactorial and can be caused by the effects of PD on the autonomic nervous system, some data indicate that levodopa administration is independently associated with orthostatic hypotension $[39,40]$. In our experience, each patient is unique with respect to the need for treatment of supine hypotension at bedtime. Providers may also recommend that patients maintain a minimum $30^{\circ}$ angle at the head of the bed to reduce the risk of supine hypertension in neurogenic orthostatic hypertension.

Weight loss should be routinely evaluated, as weight loss is a complication of treatment with LCIG as well as a marker of PD progression [41, 42]. Findings from a recent study showed that in 44 patients treated with LCIG for 51.6 \pm 28.5 (range 10-120) months, 12 experienced a $>10-\mathrm{kg}$ weight loss, and 30 patients experienced a loss of at least $2 \mathrm{~kg}$ (average weight loss, $7.6 \pm 7.1 \mathrm{~kg}$ ), with the extent of weight loss associated with the length of waking day with dyskinesia [43]. Patients can be referred to a nutritionist/ dietitian to help manage any weight loss.

Development of peripheral neuropathies has been observed with levodopa treatment, with slightly higher prevalence of peripheral neuropathy observed among patients treated with LCIG vs oral levodopa (LCIG, $42.1 \%$; oral levodopa, $30.2 \%$ ), although a causal effect has not been established $[44,45]$. The proportion of patients we see with peripheral neuropathy varies within our practices. Roughly $41 \%$ of N.K.'s patients treated with 24-h LCIG exhibit evident peripheral neuropathy, while M.J.S. and M.R. estimate only about $5-10 \%$ of their patients report neuropathy. M.J.S. and M.R. note that their patients treated with LCIG do not often complain of neuropathy, and some patients not receiving LCIG treatment do report neuropathy. A.M. has not observed a higher incidence of subacute peripheral neuropathy in patients treated with 24-h infusion, although patients with chronic neuropathy are frequently asymptomatic. As neuropathy may also be a consequence of disease progression, it may be prudent for patients receiving LCIG to be carefully monitored for the development of polyneuropathies using regular clinical and electrophysiological assessments [44]. Low levels of vitamin $B_{6}$, vita$\min \mathrm{B}_{12}$, and folate, and high levels of homocysteine and methylmalonic acid have also been correlated with peripheral neuropathy among patients treated with LCIG [45]. As with 16-h infusion, routine monitoring of vitamins $B_{6}$ and $\mathrm{B}_{12}$, folate, and homocysteine is therefore recommended for patients receiving 24-h infusion. Romagnolo et al. [45] suggested that continuous administration of intestinal gel may impede normal functioning of the intestinal mucosa, which may result in malabsorption of vitamins. Hypothetically, the likelihood of B vitamin and folate deficiencies is greater with 24 -h infusion than with $16-\mathrm{h}$ infusion, as absorption of these vitamins could be blocked for a longer time. However, data from a recent safety assessment comparing 16-h to 24-h LCIG administration indicate that the frequency of these deficiencies does not differ significantly by infusion time, and a significantly reduced frequency of polyneuropathy was observed among patients receiving 24 -h infusion ( $3 \%$ vs $21 \%, p=0.02$ ) [30]. In patients who develop subacute or chronic peripheral neuropathy, halting LCIG infusion and/or initiating vitamin supplementation, along with performing regular clinical examinations and electrophysiological measurements to monitor progression, may be necessary [44]. Furthermore, in a single patient for whom 24-h LCIG infusion induced chronic inflammatory demyelinating polyneuropathy-like neuropathy, treatment with intravenous immunoglobulin resulted in long-term improvement in neuropathy symptoms, without needing to discontinue 24-h LCIG infusion [46]. This finding may be controversial, however, as neuropathy due to vitamin deficiency stemming from LCIG treatment may not necessarily 
improve with intravenous immunoglobulin treatment, and a causal relationship between LCIG and neuropathy has not been definitively established.

Patients should also be followed for symptoms of LCIG wearing off overnight (e.g., insomnia, dystonia, sialorrhea, and pain). Changes in sleep patterns or worsening of hallucinations, delusions, and/or nightmares should also be monitored. Many of our patients receiving 24-h LCIG report improved sleep and require fewer sleep aids. However, in some patients, symptoms of psychosis (hallucinations and/or delusions) or nightmares may be exacerbated. These symptoms can be addressed by reducing the continuous LCIG dose or by prescribing an atypical anti-psychotic medication, such as pimavanserin (not available in the European Union or Australia), clozapine, or quetiapine. Quetiapine may be preferred if a quick-acting medication is required, particularly as the side effect of somnolence can assist with sleep. Pimavanserin may take at least 4-6 weeks to show effect. Some providers prefer clozapine as a last option, given the requirement for regular monitoring of bloodwork. Notably, benzodiazepines should only be used to treat rapid eye movement sleep behavior disorder, not PD-associated hallucinations or delusions. A lower infusion dose may also be helpful if patients experience dyskinesia that affects sleep quality. If patients require a greater dose during the day, providers can encourage them to use extra doses more frequently. In some patients, rapid eye movement sleep behavior disorder may be poorly controlled by 24-h LCIG only. Melatonin or clonazepam may be effective in treating the rapid eye movement sleep behavior disorder directly, along with use of an abdominal binder to protect the pump and PEG-J.

Finally, providers should discuss the potential for impulse control disorders with patients and caregivers, and monitor for the development of disorders. Although one study recorded impulse control disorders as an adverse event in four of 15 patients (27\%) treated with 16-h LCIG, other data indicate that LCIG treatment may improve symptoms of impulse control disorders [47]. Ricciardi et al. [29] reported a reduction in the frequency and severity of impulsive/ compulsive behaviors in patients treated with 24-h LCIG. Morales-Briceño et al. [30] reported that exacerbation of impulse control disorders was similar among patients treated with 16-h LCIG (two of 28 patients, 7\%) and 24-h LCIG (one of 35 patients, $3 \%$ ).

\subsection{Managing Patient Concerns}

\subsubsection{Control of Dosing}

Patients and/or caregivers may request that they have some control over medication dosing, and management of this concern is likely to be dependent on provider preference.
Different approaches may be taken, and our own strategies differ between our practices. S.T. does not allow patient control of dosing. A.M. allows patient control of dosing within the range of $0.3 \mathrm{~mL} / \mathrm{h}$. N.K. permits patients to lower their continuous dose to a pre-specified limit and notes that if the patient or caregiver can learn the logic behind changing the dose, it is generally worthwhile to teach them. V.F. allows control according to a pre-planned schedule (e.g., increasing the rate by $0.1 \mathrm{~mL} / \mathrm{h}$ every 2 or 4 weeks according to response). The pump itself can be programmed to a lock level 1 setting, which allows for the patient/caregiver to adjust the continuous dose throughout the day or night to help control motor fluctuations. The maximum rate is generally set by the clinic. M.J.S. and M.R. will use the lock level 1 setting after a transition period or if the patient lives far away. Some hospitals use lock level 0, which allows for the dosage to be adjusted freely by patients or caregivers [31]. Because patients know whether they are experiencing a good or bad day (e.g., feeling more "off" or dyskinetic), lock level 0 or lock level 1 can allow for slight dose adjustments to accommodate for day-to-day changes in mobility.

\subsubsection{Patient Acceptability}

Patients may also express concerns about comfort or moving/adjusting the equipment. Acceptance of the PEG-J port may initially be a barrier to implementing LCIG treatment. We have found that reiterating the reversible nature of the procedure and stressing that patients can always return to daytime dosing to be useful strategies in helping patients accept the PEG-J port. In addition, our experience suggests that most patients become acclimated to the therapy after a few weeks, and that the efficacy of the treatment typically outweighs the inconvenience of the pump's noise level. Many patients express that they are no longer bothered by the idea of the pump/PEG-J port after several weeks. If the sound of the pump affects the patient's insomnia, a white noise machine may be recommended. Some patients may also benefit from accessories to aid in medication delivery. Abdominal binders, for example, may be used to limit the movement of tubing and improve comfort. Patients may also find sleeping on their backs or sides, with the pump on the bed, to be more comfortable.

\subsection{Positive Effects for Patients and Caregivers}

In our clinical practices, we have anecdotally observed various positive effects of 24-h LCIG for patients and caregivers. Patients treated with 24-h LCIG have reported improved ability to sleep through the night without having to use the bathroom. Some patients also report improved sexual activity, despite initial concerns regarding comfort or displaced tubing. Some patients with PD report wearing off or freezing 
that may affect their ability to perform as desired in sexual intercourse. As a result, some patients receiving LCIG therapy choose to use an extra dose of LCIG before removing their pump for sexual intercourse. Other patients have reported improved sexual performance owing to increased muscle strength.

In our experience, the use of 16- and 24-h LCIG may potentially lead to the development of impulse control disorders, including excessive night-time eating. Patients with PD, including those treated with LCIG, frequently experience constipation, weight loss, and loss of appetite. It is unclear whether these symptoms are due to LCIG treatment or disease progression. Complications resulting from disease progression could therefore become more apparent for these patients, but may not stem from LCIG treatment itself.

We have observed that some patients and caregivers in the USA express concerns regarding the approval status for LCIG, which permits a maximum administration of 16 hours, and that this sometimes affects the decision to transition to 24-h LCIG use. Providers may reiterate that while a 16-h duration was used in the initial study to gain approval, LCIG can be used for the length of time appropriate for the patient. Furthermore, in our experience, caregivers of patients who have switched to 24-h LCIG administration have reported reduced burden of responsibility, greater happiness, improvements in their own quality of sleep, and reduced anxiety regarding the patient.

\section{Conclusions}

Infusion of LCIG for 16 hours is a standard treatment for patients with advanced PD. Although 16-h infusion is clinically indicated by the US and European Union label (and 24-h LCIG infusion is only indicated when medically necessary in the European Union), data from several small studies indicate that 24-h LCIG infusion may further reduce PD symptoms. Infusion of LCIG for 24 hours may benefit patients when reductions in nocturnal symptoms and improvements to quality of sleep are needed, chiefly when patients experience substantial wearing off of LCIG overnight, delayed morning latency, insomnia, dystonia due to wearing off of LCIG, severe night-time akinesia or bradykinesia, severe night-time pain, and/or severe night-time urinary problems. In such patients, providers may wish to first try other medications (e.g., dopamine agonists or extendedrelease oral levodopa formulations) before transitioning patients to 24-h LCIG infusion. A brief transition period to evaluate tolerance to 24-h LCIG administration may also be desired. When transitioning patients to 24-h LCIG use, adjustments to the continuous, night-time, and/or morning doses are likely necessary. Patients should be monitored for autonomic dysfunctions; symptoms when LCIG wears off overnight; changes in weight; fluctuations in plasma levels of B vitamins, folate, and homocysteine; and changes in sleep patterns or worsening of hallucinations, delusions, and/or nightmares. In our experience, 24-h LCIG infusion is generally well tolerated, with significant positive benefits to both patients and caregivers. Given these clinical observations and the small body of currently available evidence, further research into the efficacy of 24-h LCIG infusion is warranted.

Acknowledgements Medical writing support, funded by AbbVie Inc., was provided by Michelle R. Roberts, $\mathrm{PhD}$, and Kelly M. Cameron, $\mathrm{PhD}$, CMPP, of JB Ashtin, who developed the first draft based on an author-approved outline and assisted in implementing author revisions. The authors had full editorial control of the manuscript and provided their final approval of all content.

\section{Declarations}

Funding Funding of the medical writing support for the development of this manuscript was provided by AbbVie Inc. The authors received no direct compensation for their efforts. AbbVie had the opportunity to review the final manuscript, but manuscript content was solely at the discretion of the authors and reflects the opinions of the authors. The open access fee was paid by AbbVie Inc.

Conflict of interest Sandeep Thakkar has received funding/grant support from Hoag Memorial Hospital Presbyterian as medical director for the Movement Disorders Program and honoraria for consultancy from Teva, AbbVie, Medtronic, Adamas, Amneal, and Neurocrine. Victor Fung receives a salary from NSW Health and has received unrestricted research grants from the Michael J. Fox Foundation, AbbVie, and Merz. He has served on advisory boards and/or has received travel grants from AbbVie, Allergan, Cavion, Ipsen, Merz, Praxis, Segirus, Stada, Teva, and UCB, and royalties from Health Press, Ltd. Aristide Merola has received funding/grant support from the National Institutes of Health (KL2 TR001426), Lundbeck, and Abbott and honoraria for consultancy from Lundbeck, AbbVie, Abbott, and Theravance. Meredith Rollins has received consultant compensation and honoraria from AbbVie. Michael Soileau has received consultant compensation from AbbVie, Medtronic, and Sunovion, and honoraria from AbbVie, Acorda, Amneal, Lundbeck, Merz, and Teva. Norbert Kovács has received funding/grant support from the Hungarian National Research, Development and Innovation Office, National Excellence Program, University of Pécs, and Medtronic, and honoraria for consultancy from UCB, AbbVie, Abbott, Boston Scientific, Medtronic, KRKA, Teva, Boehringer Ingelheim, and GSK Pharmaceuticals.

Availability of data and material Data sharing is not applicable to this article as no datasets were generated or analyzed.

Author contributions ST, VSCF, and NK participated in the conception of the research project. ST, VSCF, AM, MR, MJS, and NK participated in the organization and execution of the project, as well as drafting, critically reviewing, and approving the manuscript for submission.

Code availability Not applicable.

Ethics approval Not applicable.

Consent to participate Not applicable. 
Consent for publication Not applicable.

Open Access This article is licensed under a Creative Commons Attribution-NonCommercial 4.0 International License, which permits any non-commercial use, sharing, adaptation, distribution and reproduction in any medium or format, as long as you give appropriate credit to the original author(s) and the source, provide a link to the Creative Commons licence, and indicate if changes were made. The images or other third party material in this article are included in the article's Creative Commons licence, unless indicated otherwise in a credit line to the material. If material is not included in the article's Creative Commons licence and your intended use is not permitted by statutory regulation or exceeds the permitted use, you will need to obtain permission directly from the copyright holder. To view a copy of this licence, visit http://creativecommons.org/licenses/by-nc/4.0/.

\section{References}

1. Olanow CW, Stern MB, Sethi K. The scientific and clinical basis for the treatment of Parkinson disease (2009). Neurology. 2009;72(21 Suppl. 4):S1-136.

2. Virhammar J, Nyholm D. Levodopa-carbidopa enteral suspension in advanced Parkinson's disease: clinical evidence and experience. Ther Adv Neurol Disord. 2017;10(3):171-87.

3. Schapira AH, Emre M, Jenner P, Poewe W. Levodopa in the treatment of Parkinson's disease. Eur J Neurol. 2009;16(9):982-9.

4. Schapira AHV, Chaudhuri KR, Jenner P. Non-motor features of Parkinson disease. Nat Rev Neurosci. 2017;18(7):435-50.

5. Sung VW, Nicholas AP. Nonmotor symptoms in Parkinson's disease: expanding the view of Parkinson's disease beyond a pure motor, pure dopaminergic problem. Neurol Clin. 2013;31(3 Suppl.):S1-16.

6. Antonini A, Poewe W, Chaudhuri KR, Jech R, Pickut B, Pirtosek $Z$, et al. Levodopa-carbidopa intestinal gel in advanced Parkinson's: final results of the GLORIA registry. Parkinsonism Relat Disord. 2017;45:13-20.

7. Antonini A, Yegin A, Preda C, Bergmann L, GLORIA study investigators and coordinators. Poewe W Global long-term study on motor and non-motor symptoms and safety of levodopa-carbidopa intestinal gel in routine care of advanced Parkinson's disease patients; 12-month interim outcomes. Parkinsonism Relat Disord. 2015;21(3):231-5.

8. Buongiorno M, Antonelli F, Camara A, Puente V, de FabreguesNebot O, Hernandez-Vara J, et al. Long-term response to continuous duodenal infusion of levodopa/carbidopa gel in patients with advanced Parkinson disease: the Barcelona registry. Parkinsonism Relat Disord. 2015;21(8):871-6.

9. Fernandez HH, Standaert DG, Hauser RA, Lang AE, Fung VS, Klostermann F, et al. Levodopa-carbidopa intestinal gel in advanced Parkinson's disease: final 12-month, open-label results. Mov Disord. 2015;30(4):500-9.

10. Fernandez HH, Vanagunas A, Odin P, Espay AJ, Hauser RA, Standaert DG, et al. Levodopa-carbidopa intestinal gel in advanced Parkinson's disease open-label study: interim results. Parkinsonism Relat Disord. 2013;19(3):339-45.

11. Juhasz A, Aschermann Z, Acs P, Janszky J, Kovacs M, Makkos A, et al. Levodopa/carbidopa intestinal gel can improve both motor and non-motor experiences of daily living in Parkinson's disease: an open-label study. Parkinsonism Relat Disord. 2017;37:79-86.

12. Lopiano L, Modugno N, Marano P, Sensi M, Meco G, Cannas A, et al. Motor outcomes in patients with advanced Parkinson's disease treated with levodopa/carbidopa intestinal gel in Italy: an interim analysis from the GREENFIELD observational study. Neurol Sci. 2016;37(11):1785-92.

13. Lopiano L, Modugno N, Marano P, Sensi M, Meco G, Solla P, et al. Motor and non-motor outcomes in patients with advanced Parkinson's disease treated with levodopa/carbidopa intestinal gel: final results of the GREENFIELD observational study. J Neurol. 2019;266(9):2164-76.

14. Martinez-Martin P, Reddy P, Katzenschlager R, Antonini A, Todorova A, Odin P, et al. EuroInf: a multicenter comparative observational study of apomorphine and levodopa infusion in Parkinson's disease. Mov Disord. 2015;30(4):510-6.

15. Olanow CW, Kieburtz K, Odin P, Espay AJ, Standaert DG, Fernandez $\mathrm{HH}$, et al. Continuous intrajejunal infusion of levodopacarbidopa intestinal gel for patients with advanced Parkinson's disease: a randomised, controlled, double-blind, double-dummy study. Lancet Neurol. 2014;13(2):141-9.

16. Slevin JT, Fernandez HH, Zadikoff C, Hall C, Eaton S, Dubow J, et al. Long-term safety and maintenance of efficacy of levodopacarbidopa intestinal gel: an open-label extension of the doubleblind pivotal study in advanced Parkinson's disease patients. J Parkinsons Dis. 2015;5(1):165-74.

17. Standaert DG, Rodriguez RL, Slevin JT, Lobatz M, Eaton S, Chatamra K, et al. Effect of levodopa-carbidopa intestinal gel on non-motor symptoms in patients with advanced Parkinson's disease. Mov Disord Clin Pract. 2017;4(6):829-37.

18. Valldeoriola F, Grandas F, Santos-Garcia D, Regidor I, Catalan MJ, Arbelo JM, et al. Long-term effectiveness of levodopa-carbidopa intestinal gel in 177 Spanish patients with advanced Parkinson's disease. Neurodegener Dis Manag. 2016;6(4):289-98.

19. Abbruzzese G, Barone P, Bonuccelli U, Lopiano L, Antonini A. Continuous intestinal infusion of levodopa/carbidopa in advanced Parkinson's disease: efficacy, safety and patient selection. Funct Neurol. 2012;27(3):147-54

20. Wang L, Li J, Chen J. Levodopa-carbidopa intestinal gel in Parkinson's disease: a systematic review and meta-analysis. Front Neurol. 2018;9:620.

21. Wirdefeldt K, Odin P, Nyholm D. Levodopa-carbidopa intestinal gel in patients with Parkinson's disease: a systematic review. CNS Drugs. 2016;30(5):381-404.

22. Chaudhuri KR, Antonini A, Robieson WZ, Sanchez-Solino O, Bergmann L, Poewe W, et al. Burden of non-motor symptoms in Parkinson's disease patients predicts improvement in quality of life during treatment with levodopa-carbidopa intestinal gel. Eur J Neurol. 2019;26(4):581-e43.

23. Zibetti M, Romagnolo A, Merola A, Priano L, Montanaro E, Angrisano S, et al. A polysomnographic study in parkinsonian patients treated with intestinal levodopa infusion. J Neurol. 2017;264(6):1085-90.

24. Cruse B, Morales-Briceno H, Chang FCF, Mahant N, Ha AD, Kim SD, et al. 24-hour levodopa-carbidopa intestinal gel may reduce troublesome dyskinesia in advanced Parkinson's disease. NPJ Parkinsons Dis. 2018;4:34

25. Nyholm D, Jansson R, Willows T, Remahl IN. Long-term 24-hour duodenal infusion of levodopa: outcome and dose requirements. Neurology. 2005;65(9):1506-7.

26. Karlsborg M, Korbo L, Regeur L, Glad A. Duodopa pump treatment in patients with advanced Parkinson's disease. Dan Med Bull. 2010;57(6):A4155.

27. Busk K, Nyholm D. Long-term 24-h levodopa/carbidopa gel infusion in Parkinson's disease. Parkinsonism Relat Disord. 2012;18(8):1000-1.

28. Chang C, Tsui DS, Mahant N, Wolfe N, Kim SD, Ha AD, et al. 24 h Levodopa-carbidopa intestinal gel may reduce falls and "unresponsive" freezing of gait in Parkinson's disease. Parkinsonism Relat Disord. 2015;21(3):317-20. 
29. Ricciardi L, Bove F, Espay KJ, Lena F, Modugno N, Poon YY, et al. 24-Hour infusion of levodopa/carbidopa intestinal gel for nocturnal akinesia in advanced Parkinson's disease. Mov Disord. 2016;31(4):597-8.

30. Morales-Briceño $\mathrm{H}$, Mahant $\mathrm{N}$, $\mathrm{Ha} \mathrm{AD}$, et al. Long-term safety and efficacy of 24-hour levodopa-carbidopa intestinal gel in Parkinson's disease. Mov Disord. 2019;34(11):1747-8.

31. Nyholm D, Adnan M, Senek M. Real-life use of levodopa/carbidopa intestinal gel in Parkinson's disease according to analysis of pump data. J Parkinsons Dis. 2020;10(4):1529-34.

32. Burack M, Aldred J, Zadikoff C, Vanagunas A, Klos K, Bilir B, et al. Implementing levodopa-carbidopa intestinal gel for Parkinson disease: insights from US practitioners. Mov Disord Clin Pract. 2018;5(4):383-93.

33. Vizcarra JA, Situ-Kcomt M, Artusi CA, Duker AP, Lopiano L, Okun MS, et al. Subthalamic deep brain stimulation and levodopa in Parkinson's disease: a meta-analysis of combined effects. J Neurol. 2019;266(2):289-97.

34. Stocchi F, Coletti C, Bonassi S, Radicati FG, Vacca L. Earlymorning OFF and levodopa dose failures in patients with Parkinson's disease attending a routine clinical appointment using Time-to-ON Questionnaire. Eur J Neurol. 2019;26(5):821-6.

35. Merola A, Zibetti M, Angrisano S, Rizzi L, Lanotte M, Lopiano L. Comparison of subthalamic nucleus deep brain stimulation and Duodopa in the treatment of advanced Parkinson's disease. Mov Disord. 2011;26(4):664-70.

36. Zibetti M, Merola A, Artusi CA, Rizzi L, Angrisano S, Reggio D, et al. Levodopa/carbidopa intestinal gel infusion in advanced Parkinson's disease: a 7-year experience. Eur J Neurol. 2014;21(2):312-8.

37. Zibetti M, Merola A, Ricchi V, Marchisio A, Artusi CA, Rizzi $\mathrm{L}$, et al. Long-term duodenal levodopa infusion in Parkinson's disease: a 3-year motor and cognitive follow-up study. J Neurol. 2013;260(1):105-14.

38. Merola A, Romagnolo A, Comi C, Rosso M, Artusi CA, Zibetti $\mathrm{M}$, et al. Prevalence and burden of dysautonomia in advanced Parkinson's disease. Mov Disord. 2017;32(5):796-7.
39. Hiorth YH, Pedersen KF, Dalen I, Tysnes OB, Alves G. Orthostatic hypotension in Parkinson disease: a 7-year prospective population-based study. Neurology. 2019;93(16):e1526-34.

40. Noack C, Schroeder C, Heusser K, Lipp A. Cardiovascular effects of levodopa in Parkinson's disease. Parkinsonism Relat Disord. 2014;20(8):815-8.

41. Bachmann CG, Trenkwalder C. Body weight in patients with Parkinson's disease. Mov Disord. 2006;21(11):1824-30.

42. Bachmann CG, Zapf A, Brunner E, Trenkwalder C. Dopaminergic treatment is associated with decreased body weight in patients with Parkinson's disease and dyskinesias. Eur J Neurol. 2009;16(8):895-901.

43. Fabbri M, Zibetti M, Beccaria L, Merola A, Romagnolo A, Montanaro E, et al. Levodopa/carbidopa intestinal gel infusion and weight loss in Parkinson's disease. Eur J Neurol. 2019;26(3):490-6.

44. Merola A, Romagnolo A, Zibetti M, Bernardini A, Cocito D, Lopiano L. Peripheral neuropathy associated with levodopa-carbidopa intestinal infusion: a long-term prospective assessment. Eur J Neurol. 2016;23(3):501-9.

45. Romagnolo A, Merola A, Artusi CA, Rizzone MG, Zibetti M, Lopiano L. Levodopa-induced neuropathy: a systematic review. Mov Disord Clin Pract. 2019;6(2):96-103.

46. Pinter D, Deli G, Juhasz A, Pal E, Janszky J, Kovacs N. Long-term successful treatment of chronic inflammatory demyelinating polyneuropathy-like polyneuropathy induced by levodopa-carbidopa intestinal gel with intravenous immunoglobulin. Eur J Neurol. 2019;26(12):e96-7.

47. Catalan MJ, Molina-Arjona JA, Mir P, Cubo E, Arbelo JM, Martinez-Martin P, et al. Improvement of impulse control disorders associated with levodopa-carbidopa intestinal gel treatment in advanced Parkinson's disease. J Neurol. 2018;265(6):1279-87. 